\title{
Improved EMG Pattern Recognition Using the Distribution Plot of Cepstrum
}

\author{
Chih-Lung Lin*, Wen-Juh Kang, Cheng-Tao Hu, Shuenn-Tsong Young, \\ Jin-Shin Lai, Maw-Huei Lee, and Te-Son Kuo \\ Department of Electrical Engineering National Taiwan University Taipei 10617,Taiwan \\ *E-mail: cllin@cc.ee.ntu.edu.tw
}

\begin{abstract}
In this paper, a real-time on-line plot is developed which recognizes user motion using the first and second cepstral coefficients for pattern recognition of the electromyogram (EMG). The cepstral coefficients, derived from autoregressive coefficients and estimated by a recursive least square algorithm, are used as the recognition features. The features are then discriminated using a modified maximum likelihood distance classifier. The cross distribution of the first and second cepstral coefficients can be plotted real-time and on-line. The physician or user can adjust the specific motions to attain optimal recognition results using this information. Subjects can be trained to contract muscles in specified and easily achievable patterns by the distribution plot. The recognition results can be used as myoelectric prosthetic control, or providing commands for the human-computer interface.
\end{abstract}

\section{INTRODUCTION}

Many tetraplegic patients have been injured around the $\mathrm{C} 5 / \mathrm{C} 6$ levels. Some of them use an automatic wheelchair with a long stick hooked in the mouth as a control. However, it is inconvenient for them to control the movement of the wheelchair in this mechanic way. Above-lesion electromyographic (EMG) signals have been utilized in functional electrical stimulation (FES) systems and artificial limbs[1-3]. Discrimination of the EMG signatures of designated motions provides a more natural way of delivering control command. The electromyogram (EMG) signal is the electrical manifestation of the activation of motor units associated with a contracting muscle. Different modes of muscle contraction are associated with various motions resulting in EMG signatures are generated. EMG signal processing using pattern recognition based on the difference in EMG signature can be used to recognize the subject's intentions.

The performance of a pattern recognition system is mainly related to the selection of the feature set and the classifier. Several strategies in functional discrimination rely on times series models of the EMG. The fourth-order autoregressive (AR) model was widely adopted and proved to have provided satisfactory experimental results before the introduction of the cepstral method. It was shown that the cepstral coefficients achieved a remarkable improvement over the conventional AR (or equivalently linear predictive coding) coefficients in the recognition of speech[4] and EMG signals[5-6].

Our previous report indicate that a higher recognition rate and the shortest amount of time were accompanied with consistent motions[5-6]. These results indicated that the recognition rate is correlated with the overlapping degree in feature space among different motions. The variance of features is related to individual controllability. For subjects with better controllability, it is easier to get consistent motions, which corresponds to small variance. Low variance in features usually leads to low degree of overlapping and high recognition rate.

In this study, the aim is to develop a real-time on-line tool to train the consistent motion of the subject. With the aid of the present method, it is easy to observe the distribution of features on-line and help the subject modify the specific motion to achieve better recognition rate.

\section{MATERIALS AND METHODS}

\section{A. Experimental methods}

Two pairs of silver surface disc electrodes (diameter 8 $\mathrm{mm}$ ) with conductive paste were bilaterally located on the right and left sides of the neck. As for each differential pair of two electrodes, one was located over the sternocleidomastoid (one-third of the muscle length distal to the insertion) and the other over the upper trapezius (onethird of the muscle length distal to the origin). A ground electrode was attached to the right earlobe. The hardware of the system was shown in Fig. 1. It contains the Gould Universal amplifier, a data acquisition and processing board (DS1 102 manufactured by DSPACE International), and an IBM compatible personal computer (PC) with an Intel 80586 processor.

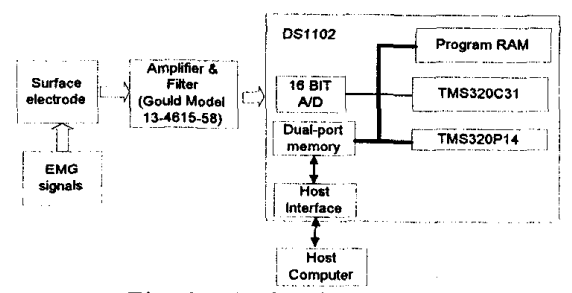

Fig. 1. The hardware block of the system 
The differential EMG signals induced on the electrode plates were fed to the Gould Universal amplifiers (Model 134615-58, input impedance $=100 \mathrm{M} \Omega, \quad \mathrm{CMRR}>70 \mathrm{~dB}$, noise $<50 \mu \mathrm{V} p-p)$. Filters in the amplifiers were set to have band-pass characteristics with a bandwidth of $10 \mathrm{~Hz}$ to $1 \mathrm{kHz}$ to minimize low-frequency movement artifacts and highfrequency noises. The amplification was ranged from 2000 to 5000. The DS1102 board contains a TMS320C31 DSP, A $128 \mathrm{~Kb}^{*} 32$ dual-port memory, analogue-to-digital (A/D) converter and digital-to-analogue (D/A) converters. The program and data transfers between host PC and TMS320C31 are through the dual-port memory configuration.

\section{B. EMG signal processing:}

The Integrated EMG (IEMG) was used to detect the muscle activity. A threshold must be included to identify the muscle contraction from background noise. The cepstral coefficients can be obtained from the linear prediction coefficients (LPC). The LPC are equivalent to the coefficients of the AR model in time-series analysis. Given the AR coefficients, $a_{i}, 1 \leq i \leq p$, the cepstral coefficients $c_{i}$ were obtained from the AR coefficients using the following recursive relationship [4]:

$c_{1}=-a_{1}$

$c_{n}=-\sum_{k=1}^{n-1}\left(1-\frac{k}{n}\right) a_{k} c_{n-k}-a_{n} \quad 1<n \leq p(1)$

The parameter $p$ is the order of the AR model. It can be determined by examining whether the residual error $\boldsymbol{e}_{\boldsymbol{k}}$ is nearly white. A four-order AR model has been widely adopted for EMG discrimination among various investigators. It had been shown that a four-order model provided the better performance in our previous study [5]. Therefore, the fourorder model is adopted in this research.

The AR coefficient $\left(a_{i}\right)$ can be estimated from the recursive least square (RLS) algorithm in sequential form. The MMLD from feature vector $X_{i}$ of unknown class to $r$ th prototype of the reference class is given as follows [7]:

$d_{M}^{2}\left(X_{i}, m_{r}\right)=\sum_{j=1}^{D} v_{j r}^{-1}\left(x_{j i}-m_{j r}\right)^{2}+\sum_{j=1}^{D} \ln v_{j r}$

where $\boldsymbol{D}$ is the feature space dimension; $X_{i}=\left|x_{1 i}, x_{2 i}, \ldots, x_{D i}\right|^{T}$ and $x_{j i}$ is the $j$ th feature coefficient of $X_{i} ; m_{r}=\left[m_{1 r}, m_{2 r}, \ldots, m_{D r}\right]^{T}$ is the mean vector of the $r$ th reference class; and $\boldsymbol{v}_{j r}$ is the variance of the $r$ th reference class in $j$ th dimension. Owing to the variations of the subjects and the electrode location, the reference feature space must be established on-line. The reference feature space $\left(m_{r}, v_{j r}\right)$ is obtained by the average of the numerous feature vectors of motions while training. The smallest MMLD was used to define the unknown motion to which a particular movement belongs. After training, the reference feature space was established online in the system RAM. According to the established reference space, the experimental motion was decided, and then the control command was identified.

\section{Experimental protocol:}

The five motions of the neck and shoulder listed in Table I were adopted because these commands could be correctly recognized by $\mathrm{C} 5 / 6$ quadriplegia. The abbreviations in Table I were designated using the following rule : region direction - action. For example, "Hlr" means that the body is Head $(\mathrm{H})$, the action direction is left (l), and the action is rotation ( $r$ ). Therefore, "Hlr" represents signature for head left rotation. Since both EMG signals from channel $R$ and channel $\mathrm{L}$ could be detected for each motion, to further analysis each signal, they would be separately represented. For example, HIrR and HIrL would respectively be used to represent the signals detected from channel $R$ and channel $L$ for the motion Hlr. It was necessary to execute a training program to improve the consistency of motion repetition for each test subject. During testing, each specific motion was repeated 5 times to establish a reference for cepstral features. The system can immediately get the cepstral coefficients of the motion and transfer these data to the computer to plot the distributions of the cepstral coefficients for these motions. The system can provide on-line help for the physician to adjust the motion of the user to get the best recognition cross distribution plot of the areas. The $\mathrm{C} 1$ and $\mathrm{C} 2$ for the five motions can be plotted on-line to provide the adjustment reference.

Table 1 Five motions performed in the experiment

right shoulder elevation (Sre)

left shoulder elevation (Sle)

head right rotation (Hrr)

head left rotation (Hlr)

both shoulders elevation (Sbe)

\section{RESULTS}

Fig. 2(a) shows the cross distribution for $\mathrm{C} 1$ and $\mathrm{C} 2$ of channel R. For shoulder motions, the areas of both shoulders elevation-channel $R$ (SbeR) and right shoulder elevationchannel $R$ (SreR) overlap, but these two areas are separated by left shoulder elevation-channel $R$ (SleR) clearly. In the motion of the head, the areas for head right rotation-channel R (HrrR) overlap with the shoulder motion SleR significantly. In Fig. 2(b), a similar result was observed in that shoulder 
left elevation-channel L (SleL) overlapped with both shoulders elevation-channel $L$ (SbeL) partly and these two areas were separated by the right shoulder elevation-channel $\mathrm{L}$ (SreL). The shoulder right elevation-channel L (SreL) also overlapped with head left rotation-channel L (HIrL)

\section{DISSCUSION}

The distributions for $\mathrm{C} 1$ and $\mathrm{C} 2$ for the normal Subject A, shown in Fig. 2, are the dominant coefficients used to define the unknown motion to which a particular movement belongs when using the smallest MMLD. The mean values for the last two cepstral coefficients (C3 and C4) are small and the variances are relatively large. According to the above description, $\mathrm{C} 3$ and $\mathrm{C} 4$ play a less important role through the MMLD discrimination. In Fig. 2(a), the areas for C1-C2 cross distribution plots of SreR and SbeR in channel $R$ are closely and partly overlapping. This interweaving is due to the fact that the motor units of right shoulder are activated in two motions, The reason these two motions were recognized correctly was that the motor units of left shoulder are activated when both shoulders elevate (SbeL), but are not activated when only the right shoulder elevates (SreL). The significant separation between SbeL and SreL in channel $\mathrm{L}$ is the dominate factor that the two motions can be classified clearly using the cepstral coefficients of two channels by MMLD classifiers. Similarly, the areas for HrrR and SleR overlap, because the motor units of right shoulder are not activated on both two motions. In Fig. 2(b) the two motions (HrrL and SleL) are separated apparently in channel L. From these results, it is apparent that the feature space of $\mathrm{Cl}$ and $C 2$ of both channels $R$ and $L$ is a powerful index for evaluating the features distribution status of control command.

\section{REFERENCES}

[1] J. S. Petrofsky, C. A. Phillipse, and H. H. Heaton, "Feedback control system for walking in man," Comput. Biol. Med., vol. 14, no. 2, pp. 135-149, 1984.

[2] G. R. Cybulski, R. D. Penn, and R. J. Jaeger, "Lower extremity neuromuscular stimulation in cases of spinal cord injury," Neurosurg., vol. 15, pp. 132-146, 1984.

[3] P.C. Doersschuk, K. E. Gustafson, and A. S. Willsky, "Upper Extremity Limb Function Discrimination Using EMG Signal Analysis,"e," IEEE Trans. Biomed. Eng., vol 30, pp. 18-28, Jan. 1983.

[4] B. S. Atal, "Effectiveness of linear prediction characteristics of the speech wave for automatic speaker identification and verification," J. Acoust. Soc. Amer. vol. 55, pp. 1304-1312, 1974.

[5] W. J. Kang, J. R. Shiu, C. K. Cheng, J. S. Lai, H.W. Tsao and T. S. Kuo, "The application of ceptral coefficients and maximum likelihood method in EMG pattern recognition," IEEE Trans. Biomed. Eng., vol 42, pp. 777-785, Aug. 1995.

[6] W. J. Kang, Jiue-Rou Shiu, Cheng-Kung Cheng, JinShin Lai, Hen-Wai Tsao, and Te-Son Kuo, "The Effect of Electrode Arrangement on Spectral Distance Measures for Discrimination of EMG Signals," IEEE Trans. Biomed. Eng., vol 44, pp. 1020-1023, October 1997.

[7] S. T. Bow, Pattern Recognition and Image Preprocessing. NY: Marcel Dekker, 1992.

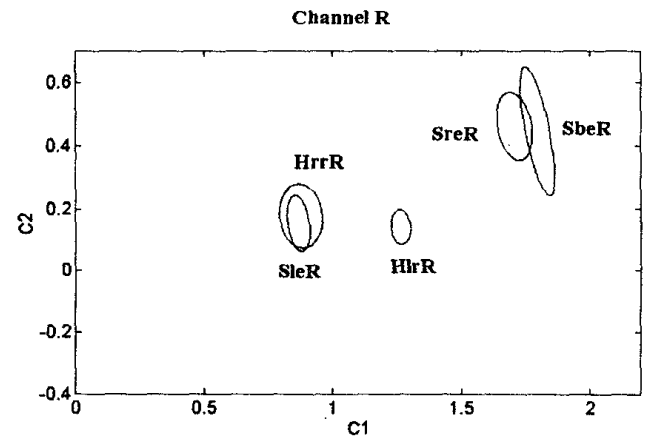

(a)

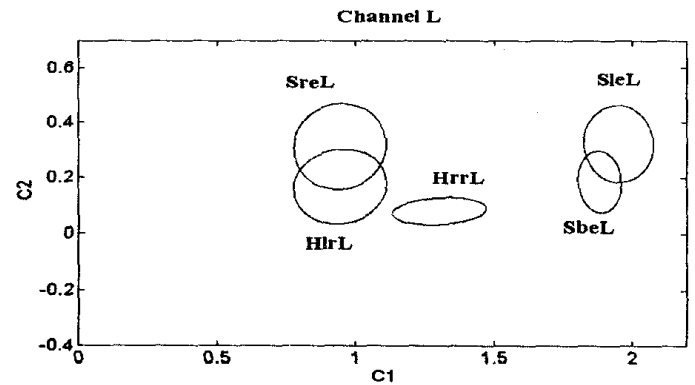

(b)

Fig. 2 The distribution of the cepstral coefficients of normal test Subject A. 